\title{
Workplace Physical Activity and Health Risks: A Need for Effective Screening and Intervention Strategies
}

\section{Dr Ahmad Alkhatib*}

Department of Sport Science Program, College of Arts and Sciences, Qatar University, Doha, Qatar

\section{Editorial}

The World Health Organisation (WHO) has adopted a global strategy on physical activity and health since 2005 , with an aim to reduce and reverse the constant rise in these health risks globally. However, most recent statistics about mortality rates caused by cardiovascular disease (CVD) are on the rise, with an estimated 17.5 million in 2012, representing $31 \%$ of all global deaths, and with an estimated 15.1 million are of these deaths due to coronary heart disease and stroke (WHO 2014) [1]. Therefore, engaging individuals, especially those who are at high CVD risk such as sedentary and obese and older populations, in a variety of exercise and physical activity patterns in order to specifically target the reduction of these risks, provides a major challenge for health professionals of this century $[2,3]$. Perhaps, identifying appropriate settings using a multifaceted approach could help in ensuring effective health and risk reduction outcomes and long-term compliance [3].

The workplace serves as an ideal setting to administer lifestyle interventions, particularly diet and physical activity interventions. Workplace interventions have been recommended because of the accessibility to a large number of employees, encouraging healthy and active lifestyle and achieving better adherence to healthy behaviours [4]. In particular, white-collar employees who spend significant amount of their weekly hours at their workplace, have been shown to benefit from a variety healthy interventions resulting in favorable reduction of several disease risk factors and consequently improved rates of health-related absenteeism, reduced health-care costs and improved productivity $[5,6]$.

Establishing an effective workplace intervention strategy requires a multidisciplinary approach that relies on effective screening and evaluation tools for assessing the prevalence and intervention outcomes. Workplace interventions have commonly relied on indirect methods to assess physical activity and healthy behaviour patterns, with tools such as the International Physical Activity Questionnaire (IPAQ), step count pedometers and accelerometers, dietary questionnaires (such as Food Frequency Questionnaire). These tools have shown various degrees of effectiveness in understanding the prevalence of certain health risk factors such as sedentriness, physical inactivity and poor diet in whitecollar workers $[7,8]$. However, technological advances can now provide occupational health specialists with more direct measurements that provide a more valid and reliable approach in designing an effective intervention, which enables targeting individual workers who may be at risk. For example, reliable assessments of blood glucose, cholesterol, body fat percentage, blood pressure, lung function, muscle strength, truck and back flexibility, and cardiorespiratory capacity, all could provide a comprehensive diagnostic tool for determining the risks of type-II diabetes, hypercholesterolemia, obesity, hypertension, and cardiorespiratory and physical capacity [9]. Results of these assessments can be measured against healthy recommended thresholds or tracked periodically throughout the intervention. Those assessments could be administered individually [10], or collectively, especially when combined with other qualitative lifestyle tools within the workplace, in order to provide a tailored reduction in the identified CVD risks, as recently been shown in within a university campus workplace $[3,9]$.
Therefore, current research evidence suggests promising risk reduction effectiveness of on-site workplace interventions, particularly when targeted at reducing specific CVD risks amongst employees at high CVD risks, and interventions that are based on a multifaceted approach such as diet and physical activity and exercise.

Understanding the barriers when conducting a workplace intervention remains a matter for ongoing research but must be considered when designing a workplace intervention, whether of physical activity and exercise alone or when exercise is combined with dietary interventions [3,11]. For example, often-reported barriers to workplace physical activity interventions, particularly the ones conducted on-site, include organisational policies such as managerial consent and flexible working hours, job demands, lack of time and energy, self-efficacy for exercise [3,12]. Similar barriers have been reported to workplace interventions aimed at adopting a healthy dietary pattern, which included work stress, lack of time for food preparation and availability of healthy food items [11]. Other reported barriers to workplace health interventions also include low participation and marketing for the intervention, shift work and overtime; those have been reported to be more apparent barriers in blue-collar workers [4].

Most workplace intervention strategies have been targeted at whitecollar workers, perhaps due to an increased reliance on technological advances in workplaces, which meant an increased number of whitecollar employees, and hence increased risk of sedentary behaviour, physical inactivity and poor nutritional habits in those employees. Future research may address the workplace intervention barriers, particularly those related to participation and adherence, in both whitecollar and blue-collar workers, especially the dietary patterns when physical activity levels appear adequate.For example, investigating the prevalence of workplace health risks and intervention barriers in low-income blue-collar workers, and migrant workers in high-income countries.

\section{References}

1. World Health Organization (WHO) (2014) Global Health Observatory (GHO) World Health Statistics.

2. Blair SN, Brodney S (1999) Effects of physical inactivity and obesity on morbidity and mortality: current evidence and research issues. Med Sci Sports Exerc 31: S646-S662.

*Corresponding author: Ahmad Alkhatib, Department of Sport Science Program, College of Arts and Sciences, Qatar University, Doha, Qatar, Tel: 0097477230662 E-mail: drahmadalkhatib@gmail.com

Received February 03, 2015; Accepted February 05, 2015; Published February 06,2015

Citation: Alkhatib A (2015) Workplace Physical Activity and Health Risks: A Need for Effective Screening and Intervention Strategies. J Health Edu Res Dev 3 : e121. doi:10.4172/2380-5439.1000e121

Copyright: (c) 2015 Alkhatib A. This is an open-access article distributed under the terms of the Creative Commons Attribution License, which permits unrestricted use, distribution, and reproduction in any medium, provided the original author and source are credited. 
Citation: Alkhatib A (2015) Workplace Physical Activity and Health Risks: A Need for Effective Screening and Intervention Strategies. J Health Edu Res Dev 3: e121. doi:10.4172/2380-5439.1000e121

Page 2 of 2

3. Alkhatib A (2015) High prevalence of sedentary risk factors amongst university employees and potential health benefits of campus workplace exercise intervention.

4. WHO (2005) Global strategy on diet, physical activity and health.

5. Quintiliani L, Sattelmair J, Sorensen G (2007) The workplace as a setting for interventions to improve diet and promote physical activity. WHO.

6. Mills PR, Kessler RC, Cooper J, Sullivan S (2007) Impact of a health promotion program on employee health risks and work productivity. Am J Health Promot 22: 45-53.

7. Musich S, Hook D, Baaner S, Edington DW (2006) The association of two productivity measures with health risks and medical conditions in an Australian employee population. Am J Health Promot 20: 353-363.

8. Clemes SA, Patel R, Mahon C, Griffiths PL ( 2014) Sitting time and step counts in office workers. Occup Med (Lond) 64: 188-192.

9. Bonauto DK, Lu D, Fan ZJ ( 2014) Obesity prevalence by occupation in
Washington State, Behavioral Risk Factor Surveillance System. Prev Chronic Dis 11:130219.

10. Alkhatib A (2013) Sedentary Risk Factors across Genders and Job Roles within a University Campus Workplace: Preliminary Study. Journal of Occupational Health 55: 218-224.

11. Lewis JE, Cutrono SE, Hodgson N, LeBlanc WG, Arheart KL, et al. (2015) Association between cardiovascular fitness and metabolic syndrome among american workers. J Occup Environ Med 57: 129-133.

12. Middleton G, Keegan R, Smith MF, Alkhatib A, Klonizakis M (2015) Implementing a Mediterranean diet intervention into a RCT: lessons learned from a non-Mediterranean based country. The Journal of Nutrition, Health and Aging.

13. Edmunds S, Hurst L, Harvey K (2013) Physical activity barriers in the workplace: An exploration of factors contributing to non-participation in a UK workplace physical activity intervention. Int J Workplace Health Man 6: 227-240. 